\title{
VIBRATION OF A DISCRETE-CONTINUOUS STRUCTURE UNDER MOVING LOAD WITH ONE OR TWO CONTACT POINTS
}

\author{
Anna Kumaniecka \\ Cracow University of Technology, Institute of Mathematics, Kraków, Poland \\ e-mail:pukumani@cyf-kr.edu.pl \\ Michat PrąciK \\ Cracow University of Technology, Institute of Applied Mechanics, Kraków, Poland \\ e-mail: mp@sparc2.mech.pk.edu.pl
}

\begin{abstract}
What is of particular importance in view of the development and operation of fast railway transport is the overhead system vibration excited by pantograph motion. The problems discussed in the paper are related to continuous system vibration under moving loads. Modelling the catenary-pantograph system is connected with motion of two subsystems, namely: continuous (contact wire) and discrete (pantograph). In the paper, the results of research on dynamical phenomena caused by the interaction between the pantograph and catenary are presented. The stiffness of the catenary wire is taken into account. The dynamical phenomena occurring in the system are described by a set of partial and ordinary differential equations. The solution to these equations has been obtained using approximate numerical methods.
\end{abstract}

Keywords: dynamics of pantograph, stiffness of catenary system

\section{Introduction}

The theoretical problem discussed in the paper is a technical problem connected with the dynamics of systems under moving loads (Bajer and Dyniewicz, 2012; Bogacz and Szolc, 1993; Fryba, 1999; Szolc, 2003). As a vibrating discrete-continuous structure, the pantograph-catenary system has been chosen for analysis.

In any high voltage electric traction system, the current needed for operating a train is collected from an overhead contact system by some form of sliding electrical contact. Such a system usually consists of a horizontal wire with which the pantograph makes continuous contact, and a catenary cable slung between the supports from which the contact wire is suspended at intervals by vertical dropper wires.

The complex behaviour of the catenary-pantograph system has been the focus of attention of many researchers for several years (Poetsch et al., 1997; Wu and Brennan, 1998). Over the last sixty years, many studies of the catenary-pantograph dynamic behaviour have been undertaken (Kumaniecka and Grzyb, 2000; Kumaniecka and Nizioł, 2000; Zhang et al., 2002).

In the past years, many researchers attempted to improve current collection quality in order to reduce wear and maintenance costs of both the overhead line and pantograph. Numerous studies on rail vehicles proved that the processes describing their dynamic state have a complex and non-periodic character (Poetsch et al., 1997; Kumaniecka, 2007). To improve the pantograph-catenary interface, it is essential to understand better the complex behaviour of this couple. The pantograph-catenary interaction at high speed is the critical factor for reliability and safety of high speed railways. The large amplitudes of transversal vibration of the messenger and contact wires can result in pantograph strip wear, loss of contact or disturbance of mutual interaction. 
With an increase in the train speed, the catenary-pantograph system with its dynamic behaviour proved to be a very important component for new train systems required to run at higher speed. This speed can be limited by the power supply through the overhead catenary system. The key point that describes the efficiency of the current collection is the contact force. The zero value of this force induces the brake in the current collection, but too large value can result in wear of the contact wire and pantograph strips.

The aim of the paper is to obtain a better understanding of the pantograph-catenary system dynamics. The emphasis of studies is placed on the description of contact loss and proper description of contact wire stiffness. A relatively simple analytical model presented in the paper is appropriate to gain physical insight into the pantograph-catenary system.

In the paper, an analytical method for calculating the response of a catenary to a uniformly moving pantograph is presented. To the authors' knowledge, the loss of contact of such a system has not been investigated so far.

The paper is organized in five Sections. Following Introduction 1, the models of the catenary-pantograph system including the contact wire, messenger wire, droppers, supporting towers and the pantograph itself are described in Section 2. In Section 3 an analytical method for calculating the response of a catenary to a uniformly moving pantograph is presented. The simulation results are given in Section 4. Final concluding remarks are formulated in Section 5.

\section{Modelling of the catenary-pantograph system}

The presented models belong to the class of continuous systems excited by a uniformly moving load. In the literature, many physical and a lot of different analytical models of the catenary-pantograph system have been proposed. Both the contact and carrying wire are one-dimensional systems. The contact and carrying cables have been modelled by infinite or non-infinite homogenous strings or Bernoulli-Euler beams. The pantograph has been modelled by an oscillator with two or four degrees of freedom. Such systems were studied in the past by a number of researchers employing different methods. A review paper describing the pantograph-catenary systems was presented by Poetsch et al. (1997) and by Kumaniecka (2007). The dynamic interaction between a discrete oscillator with four degrees of freedom and a continuous beam was also studied by Kumaniecka and Prącik (2011).

The simplified model of the catenary with one contact point introduced in the paper, shown in Fig. 1, is composed of two parallel infinitely long homogenous beams (the contact and carrying cables) connected by lumped elements (suspension rods), which are positioned equidistantly along the beams. The upper beam (carrying cable) is fixed at periodically spaced elastic supports. The lower beam (contact wire) is suspended from the upper beam by visco-elastic elements. These elements are used as a model of suspension rods. They are periodically placed at points along the beams. It is assumed that the distance between the supports of carrying cable is equal $l$ and between the droppers $2 l_{w}$. In the adopted model, the bending stiffness of the contact wire is taken into account (Wu and Brennan, 1999; Kumaniecka and Prącik, 2011).

The system in question is subjected to a concentrated force (model of pantograph), which is applied to the lower beam. This load moves along the lower beam at a constant velocity $v$.

Between the contact wire and pantograph there also appears the friction force. In the presented study, the friction force is neglected.

The physical model of the catenary with two contact points is presented in Fig. 2.

The mathematical model for a physical model of the catenary system adopted in this paper and shown in Fig. 2, was discussed in detail and presented in the monograph by Kumaniecka (2007). 
(2)

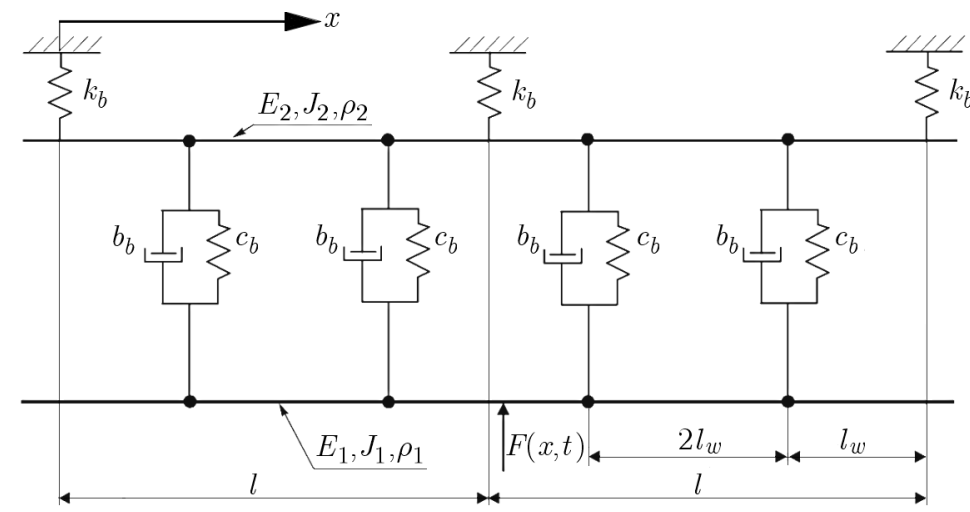

Fig. 1. Physical model of catenary with one contact point

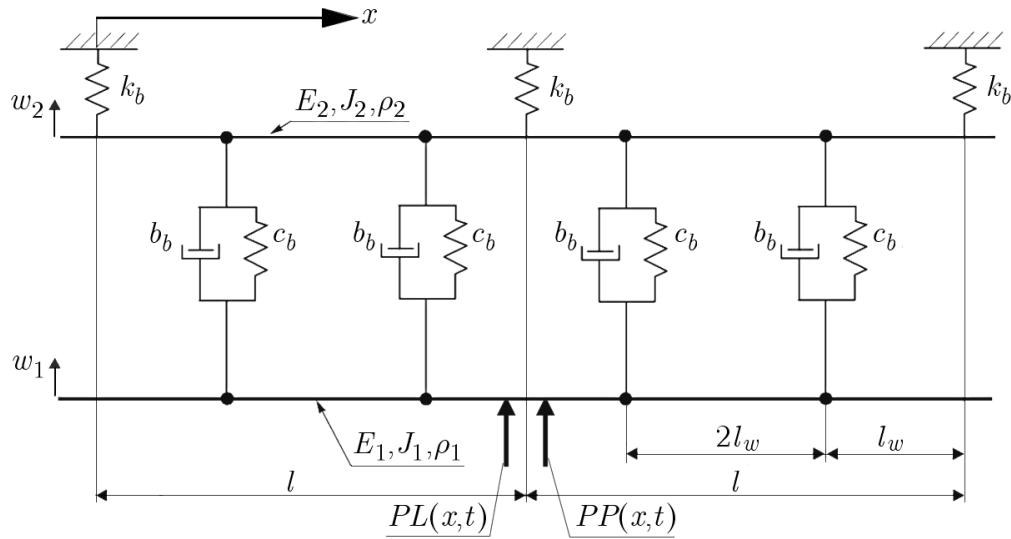

Fig. 2. Physical model of catenary with two contact points

Motion of the catenary in the vertical plane is governed by equations

$$
\begin{aligned}
& E_{1} J_{1} \frac{\partial^{4} w_{1}}{\partial x^{4}}-N_{1} \frac{\partial^{2} w_{1}}{\partial x^{2}}+\rho_{1} \frac{\partial^{2} w_{1}}{\partial t^{2}}-p+p_{F}-p_{m 1}=0 \\
& E_{2} J_{2} \frac{\partial^{4} w_{2}}{\partial x^{4}}-N_{2} \frac{\partial^{2} w_{2}}{\partial x^{2}}+\rho_{2} \frac{\partial^{2} w_{2}}{\partial t^{2}}+p-p_{m 2}=0
\end{aligned}
$$

where the following notation is used: $E_{1}, E_{2}$ - Young's modulus of lower and upper beam, respectively, $J_{i}$ - cross-sectional moment of inertia $(i=1,2), N_{i}$ - tensile force in the beams, $\rho_{i}$ - mass density, $w_{i}(x, t)$ - transversal displacements, $x$ - spatial coordinate measured along the non-deformed axis of beams, $t$ - time. The functions $w_{1}(x, t)$ and $w_{2}(x, t)$ describe the lower and upper beam transversal displacements, respectively.

The loads $p(x, t)$ acting on the beams and caused by internal forces in the springs and damping elements are treated as continuous. They can be expressed in the form

$$
p(x, t)=\sum_{(n)}\left\{c_{b}\left[w_{2}(x, t)-w_{1}(x, t)\right]+b_{b}\left[\dot{w}_{2}(x, t)-\dot{w}_{1}(x, t)\right]\right\} \delta\left(x-x_{n}\right)
$$

where: $c_{b}$ is the coefficient of spring elasticity, $b_{b}$ - damping coefficient, $x_{n}$ - coordinates of droppers spacing (concentrated masses), $x_{n}=2 l_{w}(2 s-1), s \in N, 2 l_{w}$ - distance between the droppers, $\delta$ - Dirac's function.

The interaction force between the pantograph and contact wire $p_{F}$ can be described by the term

$$
p_{F}(x, t)=F(t) \delta(x-v t)
$$


The reaction force $p_{m i}(i=1,2)$ that comes from concentrated masses $m$ spaced on the lower and upper beams acting at points $x_{n}$ can be treated as distributed and written in the form:

- for the lower beam

$$
p_{m 1}(x, t)=\sum_{(n)} m \ddot{w}_{1}(x, t) \delta\left(x-x_{n}\right)
$$

- for the upper beam

$$
p_{m 2}(x, t)=\sum_{(n)} m \ddot{w}_{2}(x, t) \delta\left(x-x_{n}\right)
$$

The boundary and initial conditions adopted for numerical simulation have been based on the assumed vibration model of a linear system (data from identification research).

In the present paper, the pantograph has been modelled as an oscillator with four degrees of freedom. The model refers to a real system designed by engineers from Schunk Wien GmbH. The basic pantograph is the standard WBL- $85 / 3 \mathrm{kV}$. The collector strips are represented by masses $\mathrm{m} 1 \mathrm{~L}$ and $\mathrm{m} 1 \mathrm{P}$, the equivalent masses of the frames are denoted by $m_{2}$ and $m_{3}$. The masses are connected by springs $c_{11}$ and $c_{22}$ to provide a nominally constant uplift force. The aerodynamic force is taken into account (Bacciolone et al., 2005). The physical model of the pantograph investigated in our studies is shown in Fig. 3.

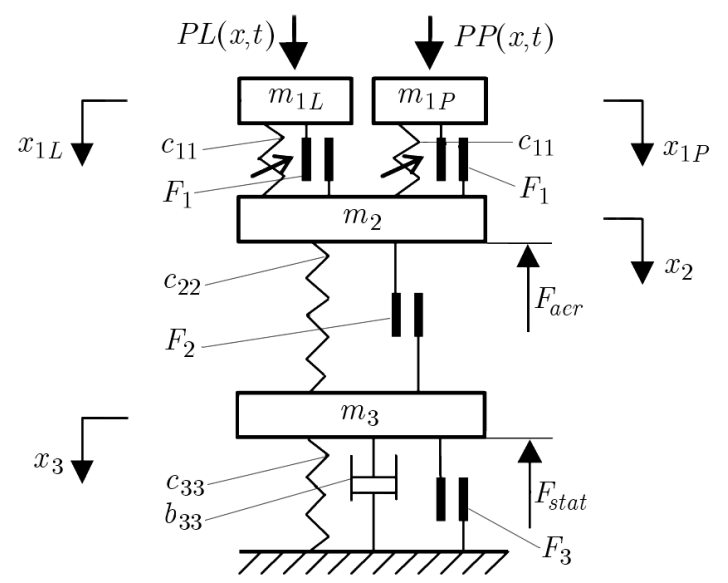

Fig. 3. Model of pantograph

The mathematical model for a physical model of the pantograph adopted in this paper and shown in Fig. 3 was discussed in detail and presented in the monograph by Kumaniecka (2007). In many real pantograph systems, the springs are guided in telescopic sliders, which gives reasons to apply dry friction elements in the physical model. The structure of the simulation model has been based on the formal notation of motion in form of ordinary differential equations.

Motion of the pantograph in the vertical plane is governed by equations

$$
\begin{aligned}
& m_{1 L} \ddot{x}_{1 L}=m_{1 L} g-\left|F_{1}\right| \operatorname{sgn}\left(\dot{x}_{1 L}-\dot{x}_{2}\right)-c_{11}\left(x_{1 L}-x_{2}\right)+P L(x, t) \\
& m_{1 P} \ddot{x}_{1 P}=m_{1 P} g-\left|F_{1}\right| \operatorname{sgn}\left(\dot{x}_{1 P}-\dot{x}_{2}\right)-c_{11}\left(x_{1 P}-x_{2}\right)+P P(x, t) \\
& m_{2} \ddot{x}_{2}=m_{2} g-\left|F_{2}\right| \operatorname{sgn}\left(\dot{x}_{2}-\dot{x}_{3}\right)+\left|F_{1}\right|\left[\operatorname{sgn}\left(\dot{x}_{1 P}-\dot{x}_{2}\right)+\operatorname{sgn}\left(\dot{x}_{1 L}-\dot{x}_{2}\right)\right] \\
& \quad-c_{22}\left(x_{2}-x_{3}\right)+c_{11}\left(x_{1 P}-x_{2}\right)+c_{11}\left(x_{1 L}-x_{2}\right)-F_{a e r} \\
& m_{3} \ddot{x}_{3}=m_{3} g-c_{33} x_{3}-b_{33} \dot{x}_{3}-\left|F_{3}\right| \operatorname{sgn}\left(\dot{x}_{3}\right)-F_{\text {stat }}+c_{22}\left(x_{2}-x_{3}\right)+\left|F_{2}\right| \operatorname{sgn}\left(\dot{x}_{2}-\dot{x}_{3}\right)
\end{aligned}
$$

where: $x_{1}, x_{2}, x_{3}, \dot{x}_{1}, \dot{x}_{2}, \dot{x}_{3}, m_{1}, m_{2}, m_{3}$ are displacements, velocities and masses of the elements, respectively, $F_{1}, F_{2}, F_{3}$ - friction forces, $F_{\text {aer }}, F_{\text {stat }}$ - aerodynamic and static forces, 
$P L(x, t), P P(x, t)$ - excitation forces, interaction forces between the pantograph and contact wire.

The displacement of the pan-head is the main factor for dynamic performance of the pantograph, and it is related to the contact forces directly.

\section{Analytical model of the system vibration}

The catenary motion can be described by means of partial differential equations (2.1), which govern small vertical vibrations of each beam in the vicinity of their equilibrium state, induced by the transversal force moving along the lower beam. Some details are presented in the monograph by Kumaniecka (2007) and in the paper by Kumaniecka and Prącik (2011). The mathematical model for a physical model of the pantograph was discussed by Prącik and Furmanik (2000).

The interaction between the pantograph and contact wire is limited to a set of two parallel forces

$$
P L(x, t) \delta(x-v t) \quad P P(x, t) \delta\left(x-v t+x_{L P}\right)
$$

In the above equation, $x$ denotes the spatial horizontal co-ordinate, $t$ time, $x_{L P}$ is the distance between the shoes.

In the case of one contact point, the function describing the contact force can be expressed as

$$
F(x, t)=P L(x, t)+P P(x, t)
$$

According to the results obtained by the authors (2011) and others (Wu and Brennan, 1999), the forces $P L(x, t), P P(x, t)$ can be connected with harmonic changes of catenary stiffness and given in the form

$$
\begin{aligned}
& P L(x, t)=k_{0}\left\{\begin{array}{lll}
{\left[1-\alpha \cos \left(\frac{2 \pi v}{L} t\right)\right]\left(x_{1 L 0}-x_{1 L}\right)} & \text { for } & x_{1 L 0}>x_{1 L} \\
0 & \text { for } & x_{1 L 0} \leqslant x_{1 L}
\end{array}\right. \\
& P P(x, t)=k_{0} \begin{cases}{\left[1-\alpha \cos \left(\frac{2 \pi}{L}\left(v t+x_{L P}\right)\right)\right]\left(x_{1 P 0}-x_{1 P}\right)} & \text { for } \quad x_{1 P 0}>x_{1 P} \\
0 & \text { for } \quad x_{1 P 0} \leqslant x_{1 P}\end{cases}
\end{aligned}
$$

In equations (3.3), the following notation is used: $x_{1 L}, x_{1 P}$ denote vertical displacements of the pantograph contact shoes, $x_{1 L 0}, x_{1 P 0}$ - vertical displacements of two points on the wire that are in contact with masses $m_{1 L}$ and $m_{1 P}$, respectively, $k_{0}, \alpha$-are stiffness coefficients (Wu and Brennan, 1999), given by formulae

$$
k_{0}=\frac{k_{\max }+k_{\min }}{2} \quad \alpha=\frac{k_{\max }-k_{\min }}{k_{\max }+k_{\min }}
$$

where $L$ is the length of one span and $k_{\max }, k_{\min }$ are the largest and the smallest stiffness values in the span, respectively.

When the model with two contact points is investigated, the contact forces are written as

$$
\begin{aligned}
& P L(x, t)=k_{0}\left\{\begin{array}{lll}
{\left[1-\alpha \cos \left(\frac{2 \pi v}{l} t\right)\right]\left[x_{1 L 0}-w_{1}(x, t)\right]} & \text { for } \quad x_{1 L 0}>w_{1}(x, t) \\
0 & \text { for } \quad x_{1 L 0} \leqslant w_{1}(x, t)
\end{array}\right.
\end{aligned}
$$

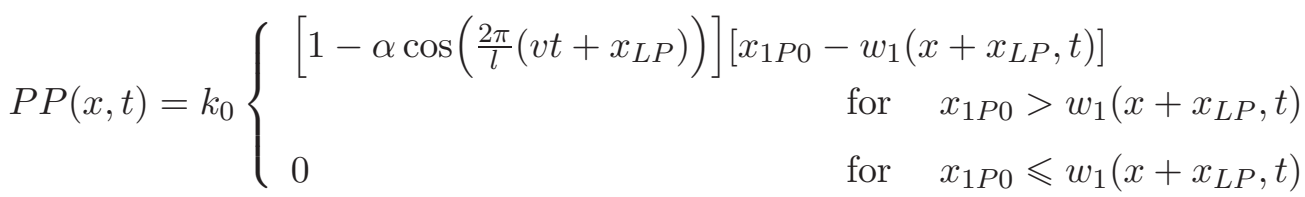


where $w_{1}(x, t)$ is the transversal displacement of the lower beam, $x_{1 L}, x_{1 P}$ are coordinates of strips motion (see Fig. 3), $x_{1 L} \equiv w_{1}(x, t) \delta(x-v t)$ and $x_{1 P} \equiv w_{1}\left(x+x_{L P}, t\right) \delta\left(x-v t+x_{L P}\right)$.

The equations of catenary motion model (2.1) are presented in the monograph by Kumaniecka (2007). After substituting relations (3.3) or (3.5), in the case of one or two contact points, respectively, to the equations of motion, they include some parameters associated with stiffness of the contact wire $k_{0}, \alpha$.

In the case of one contact point, the solutions to set of equations (2.1) have been taken in the form of waves

$$
\begin{aligned}
& w_{1}(x, t)=\sum_{p_{1}} \sum_{r_{1}} A_{r_{1}} \frac{\sin \frac{x-v t}{p_{1} l_{w}}}{\frac{x-v t}{p_{1} l_{w}}} \sin \left(\omega_{r_{1}} t-\varphi_{r_{1}}\right) \\
& w_{2}(x, t)=\sum_{p_{2}} \sum_{r_{2}} A_{r_{2}} \frac{\sin \frac{x-v t}{p_{2} l_{w}}}{\frac{x-v t}{p_{2} l_{w}}} \sin \left(\omega_{r_{2}} t-\varphi_{r_{2}}\right)
\end{aligned}
$$

where $p_{i}$ are associated with the moving modes and $r_{i}$ with the standing modes for $i=1,2$, and the coefficients $A_{r_{1}}, A_{r_{2}}$ could be determined numerically using a collocation method.

To solve equations of motion (2.1) and (2.6) for two contact points, it is necessary to employ another expression for functions which describe transversal displacements of the lower beam

$$
w_{1}(x, t)=\sum_{p_{1}} \sum_{r_{1}} A_{r 1} \frac{\sin \frac{x-v t}{p_{1} l_{w}}}{\frac{x-v t}{p_{1} l_{w}}} \sin \left(\omega_{r_{1}} t-\varphi_{r_{1}}\right)+\sum_{p_{1}} \sum_{r_{1}} B_{r 1} \frac{\sin \frac{x+x_{L P}-v t}{p_{1} l_{w}}}{\frac{x+x_{L P}-v t}{p_{1} l_{w}}} \sin \left(\omega_{r_{1}} t-\varphi_{r_{1}}\right)
$$

\section{Numerical analysis}

On the basis of the given mathematical model, a simulation program applying the package VisSim Analyze ver. 3.0 has been built. Numerical simulations have been carried out for different data sets.

The numerical calculations have been done for the following parameters of the system

$$
\begin{array}{lll}
m_{1 L}=m_{1 P}=7.93 \mathrm{~kg} & m_{2}=8.73 \mathrm{~kg} & m_{3}=10.15 \mathrm{~kg} \\
F_{1}=2.0 \mathrm{~N} & F_{2}=F_{3}=2.5 \mathrm{~N} & F_{\text {aer }}=30.0 \mathrm{~N} \\
F_{\text {aer }}=30 \mathrm{~N} & c_{3}=60 \mathrm{Ns} / \mathrm{m} & x_{L P}=1.0 \mathrm{~m}
\end{array} \quad F_{\text {stat }}=600 \mathrm{~N}
$$

The parameters of the system correspond to the parameters of the real overhead power lines for high speed trains (data set for pantograph WBL $85-3 \mathrm{kV} / \mathrm{PKP}$ ).

The block schemes of simulation of mass displacements and contact forces of the pantograph model at $F_{\text {stat }}=600 \mathrm{~N}$ and $500 \mathrm{~N}, F_{\text {aer }}=30 \mathrm{~N}$ are presented in Figs. 4 and 5 .

To investigate the phenomena of contact loss in the overhead system, numerical simulations for the contact force less than the limit force were carried out by Kumaniecka and Pracik (2011).

In Fig. 6, the loss of contact between the pantograph and catenary is illustrated.

The results of simulations of the variability of contact forces of the pantograph (with two contact points), when the vertical displacement amplitude between the contact points spaced by $1 \mathrm{~m}$, is equal $0.03 \mathrm{~m}$, are presented in Fig. 7. The simulations have been done for velocity $v=55.55 \mathrm{~m} / \mathrm{s}$ using the blockscheme of simulation similar to that presented in Fig. 4 but utilizing a different expression for $w_{1}(x, t)$ (respectively to equation (3.7)).

Based on the results of simulations, we can conclude that the pantograph with two contact strips guarantees better interaction (strips are detached convertible).

The analysis of the simulation results (see Fig. 7) shows that the response of the system in question is not harmonic, it consists of standing and moving modes. To conclude, it can be 


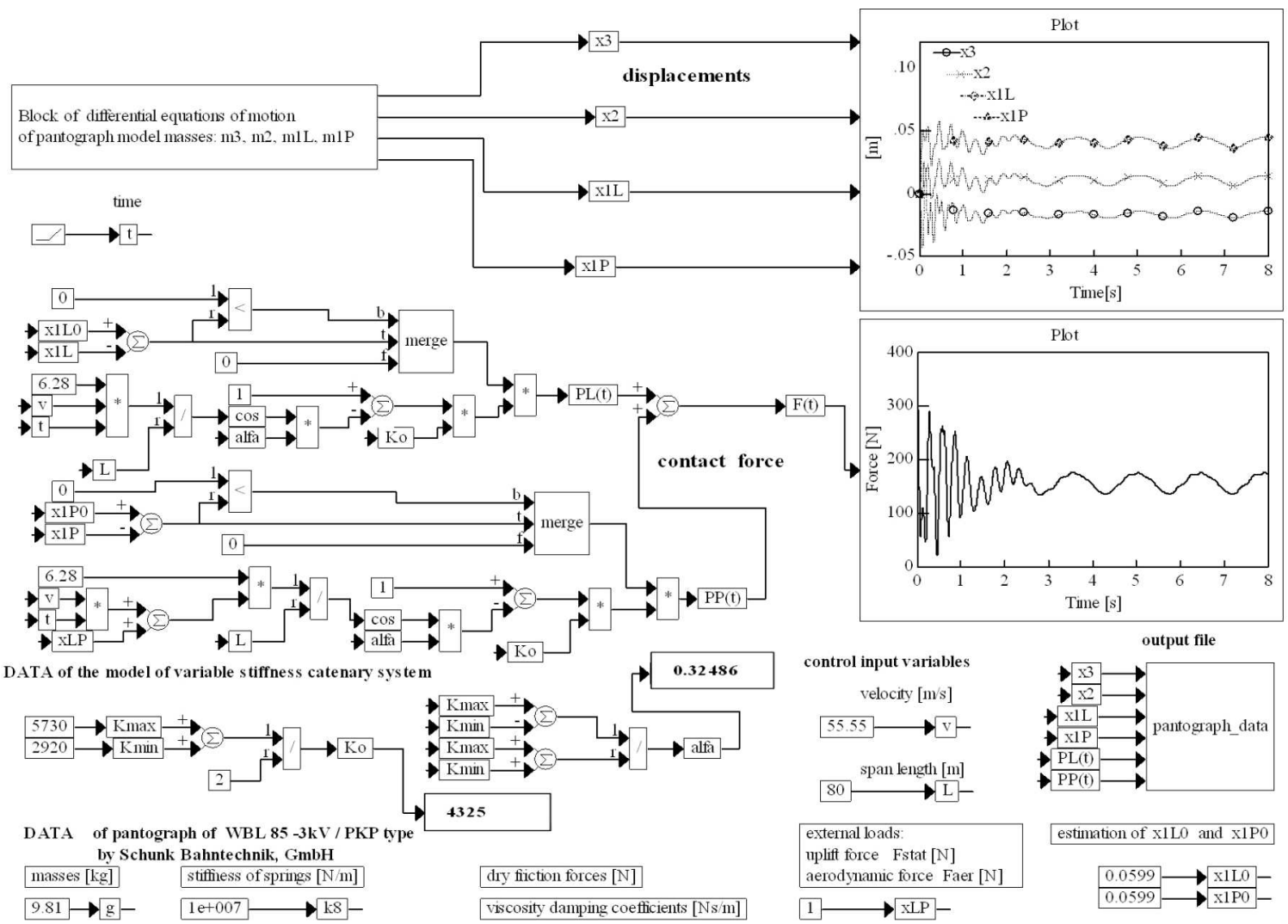

Fig. 4. Block scheme of simulation of mass displacements and contact forces of the pantograph model at $F_{\text {stat }}=600 \mathrm{~N}, F_{\text {aer }}=30 \mathrm{~N}$; in the case of one contact point, when $x_{1 L} \cong x_{1 P}$

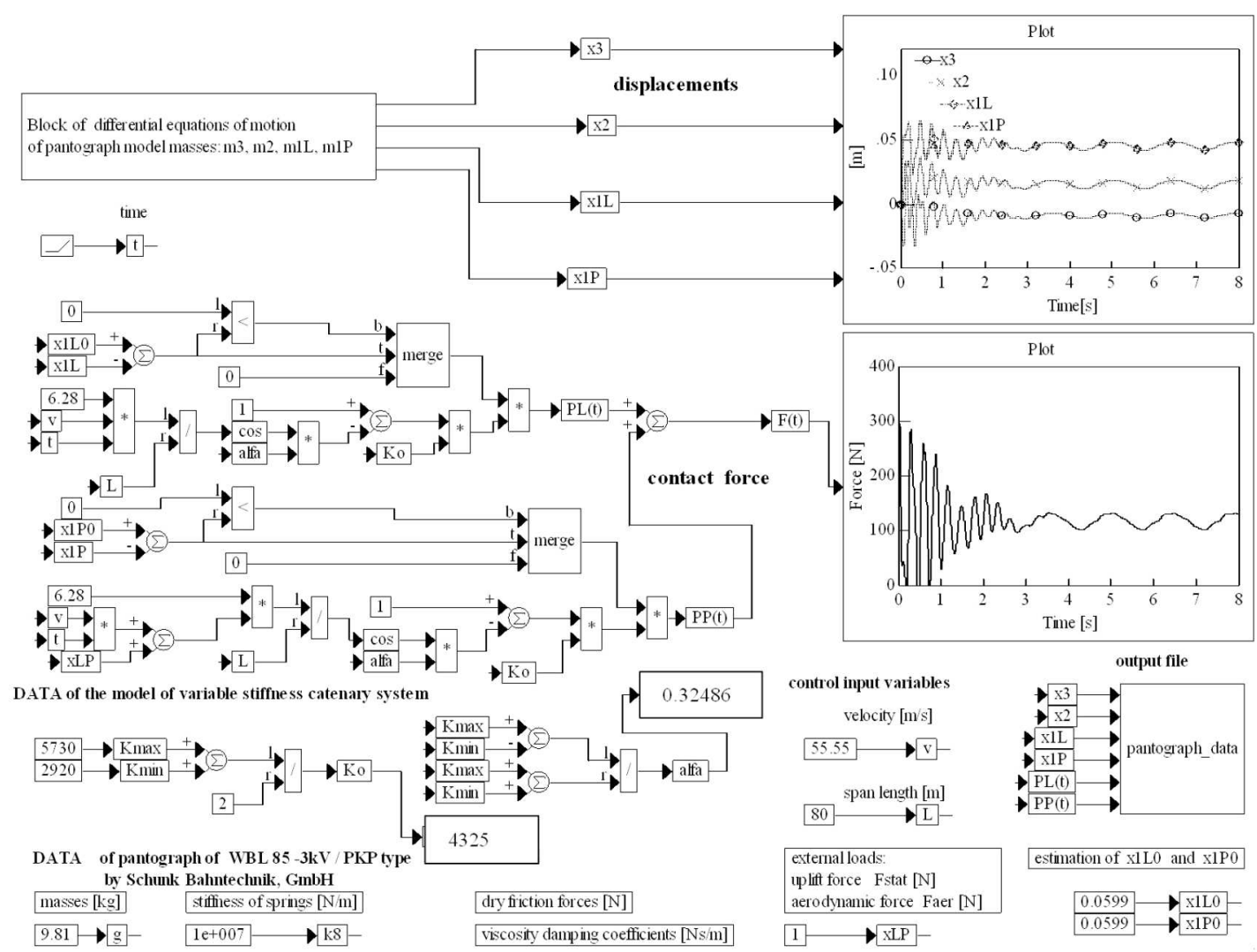

Fig. 5. Block scheme of simulation of contact forces and contact loss at $F_{\text {stat }}=500 \mathrm{~N}, F_{\text {aer }}=30 \mathrm{~N}$; in the case of one contact point, when $x_{1 L} \cong x_{1 P}$ 


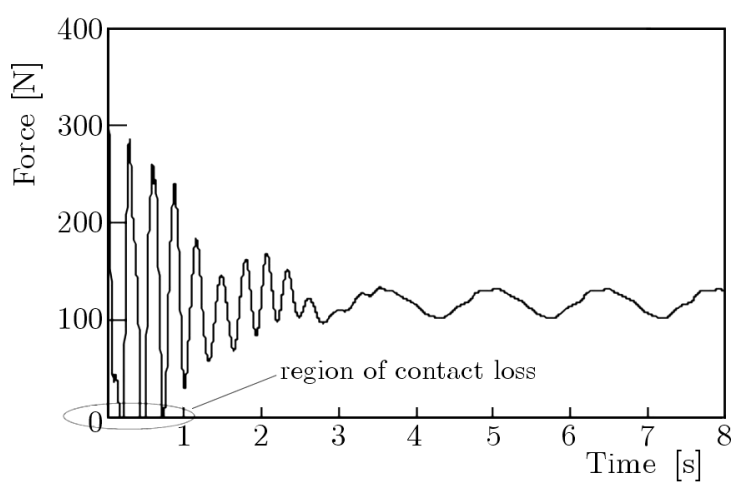

Fig. 6. Contact force for uplift force $F_{\text {stat }}=500 \mathrm{~N}$; loss of contact in the case of one contact point

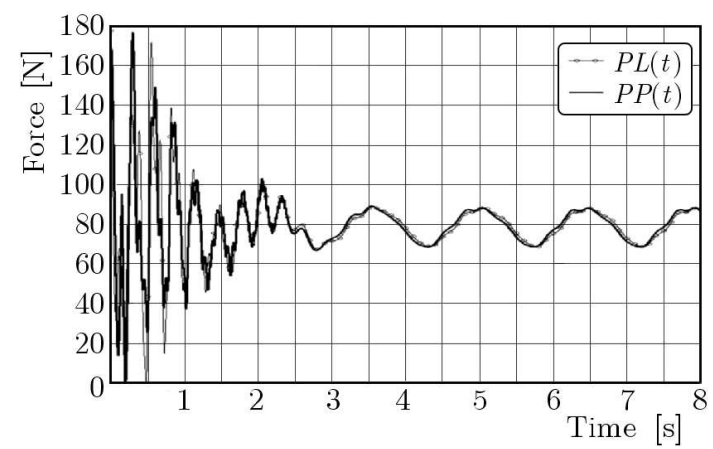

Fig. 7. Variability of contact forces of the pantograph with two contact points

stated that the motion of the contact wire has a wavy character. The calculations have confirmed that the travelling force is a source of waves propagating leftwards and rightwards at different frequencies (Snamina, 2003; Bogacz and Frischmuth, 2013).

The domination of lower frequency modes is visible (see Fig. 8). The same effect is visible in the case of a pantograph with one contact point. For a two contact points pantograph, the modal damping is more effective.

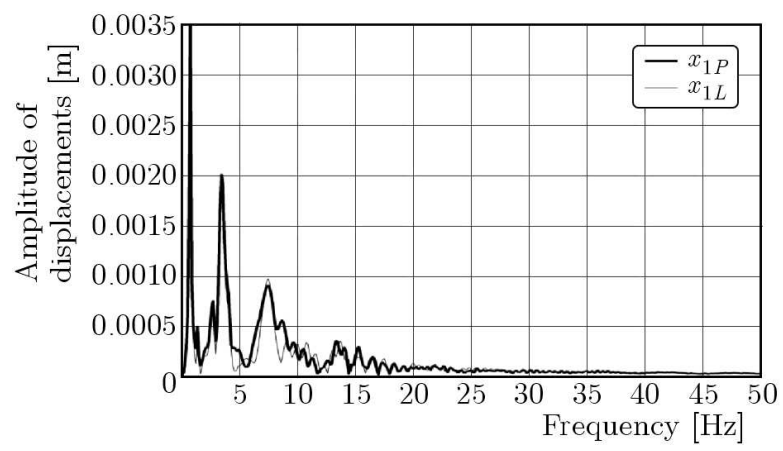

Fig. 8. Spectrum FFT of vibration displacements

In Figs. 9 and 10, some results of the lower beam vibration in the case of one or two contact points are shown. As can be seen in Fig. 10, the critical amplitude value of vibration displacement, critical as referred to displacement values $x_{1 L 0}=x_{1 P 0}=0.03 \mathrm{~m}$ adopted as an example (for the data set taken for numerical simulations at the velocity $v=55.55 \mathrm{~m} / \mathrm{s}$ ), has been exceeded.

The examples of the results presented above have been obtained with the catenary stiffness parameters and the velocity of pantograph motion $v=55.55 \mathrm{~m} / \mathrm{s}$. At higher velocities taken for 


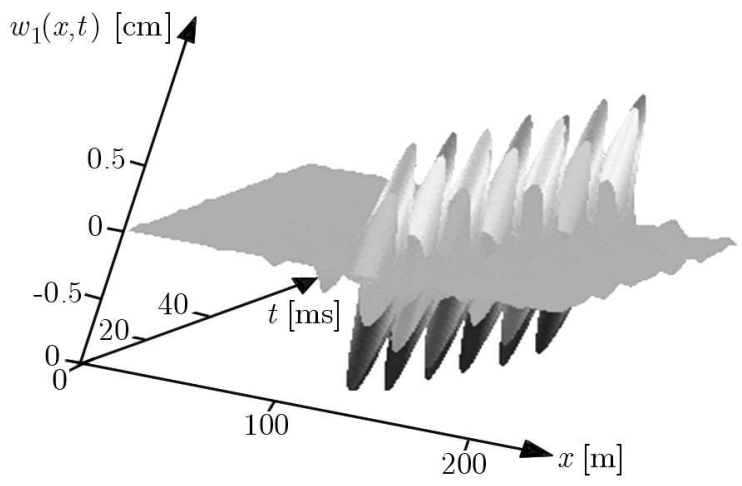

Fig. 9. Results of simulations of the beam displacement function $w_{1}(x, t)$ for $p_{1}=1, r_{1}=1,2$; in the case of one contact point

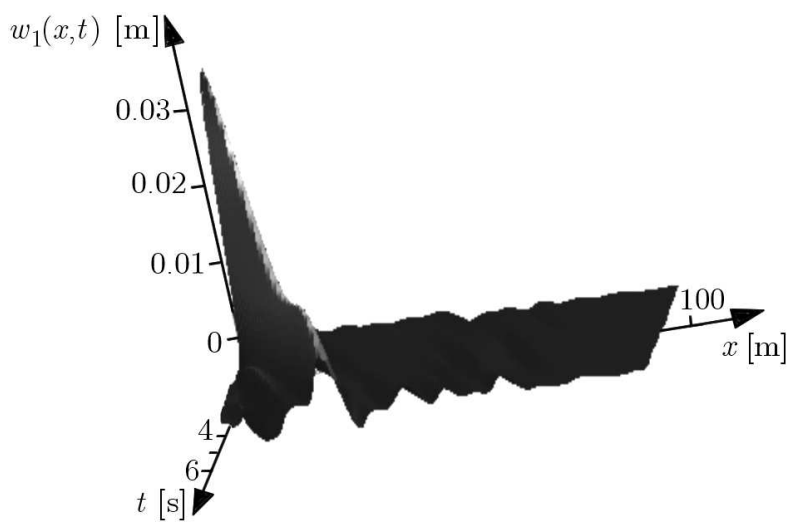

Fig. 10. Simulations of lower beam vibration of the catenary model in the case of two contact points

simulations and the analysis of subsequent excited mode vibration frequencies of the catenary in 3D graphs of displacements. there can be seen a more marked interaction in the case of two contact points pantograph. Also two maxima and minima of waves moving parallel, positioned at an angle to the time axis, are visible.

\section{Final conclusions}

The state-of-the-art of the theoretical and experimental investigations indicates the need for continuation of the research to improve the modelling of the catenary-pantograph system. In the present paper, a simplified model of the pantograph and catenary has been proposed. The equations of motion are based on a beam model with one or two concentrated varying forces moving along the contact wire at a constant velocity. The structure of the simulation model is based on a formal notation of motion in form of partial and ordinary differential equations. For the simulation, software package VisSim has been applied. The paper has discussed the application of the stiffness formula to the analysis of pantograph-catenary interaction.

On the basis of the results of simulations of the lower beam displacements (Figs. 9 and 10) the following conclusions can be drawn:

- The wave sequence is associated with the pantograph motion (the compound of three modes of moving waves and three standing ones). The frontal maximum and reverse minimum of the displacement are visible. The ratio of the absolute value of the maximum to that of the minimum is equal to 9 . 
- Damping of the displacement amplitude excited in the lower beam is time and space variable. For example, a reduction of the beam displacement maximum by about $20 \mathrm{~dB}$ occurs after approximately $0.6 \mathrm{~s}$, in the case of analysis on the span of length of about $100 \mathrm{~m}$.

The results of analysis of the simulation performed on the adopted model of mutual interaction between the pantograph and catenary have shown the domination of lower frequencies components in the spectrum of the lower beam vibration displacements, similarly to the case of one contact point system (Fig. 8). This fact has been also indicated by others scientists (Poetsch et al., 1997; Szolc, 2003). On the basis of the simulation results of the lower beam displacements, it can be concluded that there is a wave sequence associated with the pantograph motion. Damping of the lower beam vibrations, caused by the moving pantograph, is variable in time and space. The described phenomena should be taken into account in real applications in high speed railways.

\section{References}

1. Bajer C.I., Dyniewicz B., 2012, Numerical Analysis of Vibrations of Structures under Moving Inertial Load, Springer

2. Bocciolone M., Collina A., Resta F., Rocchi D., Tosi A., 2005, Pantograph aerodynamics effects on the pantograph-catenary interaction, Proceedings of XIX IAVSD Symposium, Millano, paper No. 55

3. Bogacz R., Frischmuth K., 2013, Wave propagation in catenaries subjected to moving loads, ICEDyn/2013/Sesimbro, K07, 8p

4. Bogacz R., Szolc T., 1993, Analysis of dynamic interaction between the continuous string and moving oscillator, Engineering Transactions, 41, 3/4, 361-380

5. FrYBA L., 1999, Vibration of Solid and Structure Under Moving Loads, Telford, London

6. KumanieckA A., 2007, Methods of analysis of discrete-continuous systems vibrations and their applications to the railway transport problems, Monograph, Mechanics, 352, Cracow

7. Kumaniecka A., Grzyb A., 2000, On dynamics of catenary-pantograph system modelled as discrete-continuous system, Machine Dynamics Problems, 24, 1, 105-119

8. Kumaniecka A., NizioŁ J., 2000, Dynamical phenomena in catenary-pantograph system, Scientific Issue of ATR Bydgoszcz, Mechanics, 228, 47, 15-26

9. KumanieckA A., PrĄCIK M., 2003, Modelling and identification of catenary-pantograph system, Journal of Theoretical and Applied Mechanics, 41, 4, 887-901

10. Kumaniecka A., Prącik M., 2011, Modelling of the catenary-pantograph system, Machine Dynamics Research, 35, 4, 74-91

11. Kumaniecka A., Snamina J., 2005, Transient response of the pantograph-catenary system to impulsive loads, Machine Dynamics Problems, 29, 2, 91-105

12. Poetsch G., Evan J., Meisinger R., Kortum W., Baldauf W., Veitl A., WallascheK J., 1997, Pantograph/catenary dynamics and control, Vehicle System Dynamics, 28, 159-195

13. Prącik M., Furmanik J., 2000, Problems of modelling and simulation of catenary and pantograph cooperation, Proceedings of VII-th Workshop PTSK, Warsaw, 7, 242-250

14. Snamina J., 2003, Mechanical wave phenomena in overhead transmission lines, Monograph, Mechanics, 287, Cracow

15. SzolC T., 2003, Dynamic analysis of complex discrete-continuous mechanical systems, Polish Academy of Sciences - IFTR Reports, 2/2003, D.Sc. dissertation 
16. Wu T.X., Brennan M.J., 1998, Basic analytical study of pantograph-catenary system dynamics, Vehicle System Dynamics, 30, 443-456

17. Wu T.X., Brennan M.J., 1999, Dynamic stiffness of a railway overhead wire system and its effect on pantograph-catenary system dynamics, Journal of Sound and Vibration, 219, 483-502

18. Zhang W., Mei G., Wu X., Shen Z., 2002, Hybrid simulation of dynamics for the pantograph-catenary system, Vehicle System Dynamics, 38, 6, 393-414

Manuscript received November 29, 2013; accepted for print May 11, 2015 